\title{
Resection of a left insular cavernoma aided by a simple navigational tool
}

\author{
Technical note
}

\author{
Vincenzo Esposito, M.D., Sergio Paolini, M.D., and Roberta Morace, M.D. \\ Department of Neurosurgery, "La Sapienza" University of Rome; and Department of Neurosurgery, \\ University of Perugia, Neuromed Institute, Pozzilli, Italy
}

\begin{abstract}
$\checkmark$ The management of cavernous malformations of the brain is markedly influenced by the location of the lesions themselves. In the last decade, resection of cavernomas arising in the dominant insular lobe has been deemed safe only with the guidance of neuronavigation. Most navigation equipment, however, shares some minor drawbacks, including costs, longer operating time, and a variable loss of accuracy due to intraoperative brain shift. In this paper the authors present the case of a left dominant insular cavernoma that was successfully removed using a novel form of navigation that they call magnetic resonance imaging-based corticotopography. This technique, which is unaffected by the brain shift phenomenon, provided a simple and inexpensive alternative to standard neuronavigation. Selected cases of subcortical brain lesions could be conveniently approached using the same technique.
\end{abstract}

\section{KEY WORDS - cavernoma - neuronavigation - three-dimensional reconstruction • cortex - insula}

$\mathrm{S}$ UPRATENTORIAL cavernomas are infrequently located in the insular lobe. ${ }^{1,3}$ Particularly on the dominant side, the natural history of these lesions must be carefully balanced with the surgical morbidity involved in their resection. ${ }^{2}$ Surgical approaches to the insula have been well known to neurosurgeons for decades, ${ }^{6,9,11,14}$ but they still pose significant risks. These risks have been related primarily to a wide dissection of the sylvian fissure requiring manipulation of the lenticulostriate arteries and to injury to the nearby internal capsule. ${ }^{4}$

The advent of neuronavigation systems has broadened the surgical indications for these challenging lesions. As a result, image-guided resection of insular cavernomas has been reported with increasing frequency. Tirakotai, et al., ${ }^{13}$ reported a series of eight cavernous angiomas of the insular lobe that were successfully removed with the aid of neuronavigation. Duffau and Fontaine ${ }^{7}$ coupled the use of neuronavigation with intraoperative language mapping in the awake patient to remove a cavernous angioma of the left insula.

In the case reported here we removed a cavernous angioma of the left dominant insula with the aid of a novel form of neuronavigation, which we call MR imagingbased corticotopography. We developed this simple and inexpensive technique in 2002 and have used it with

Abbreviations used in this paper: $\mathrm{CM}=$ cavernous malformation; $\mathrm{MR}=$ magnetic resonance; $3 \mathrm{D}=$ three-dimensional. increasing frequency to resect subcortical lesions lying up to $3 \mathrm{~cm}$ below the brain surface.

\section{Illustrative Case}

History and Examination. This 34-year-old woman with a 1-year history of recurrent headache was admitted to our institute after left insular CM was diagnosed based on results of MR imaging (Fig. 1). The lesion measured approximately $11 \mathrm{~mm}$, was located at a depth of $21 \mathrm{~mm}$, and was surrounded by a typical hemosiderin-stained rim on $\mathrm{T}_{2}$-weighted images. The patient had also undergone a functional MR imaging study, which demonstrated cortical activation for language tasks on the left frontal opercular region. On admission, the patient was neurologically intact. We decided to remove the lesion by using an image-guided procedure that we had recently developed as a navigation tool for subcortical targets.

Operation. The procedure entailed a 3D rendering of MR imaging volumetric sequences acquired before and after intravenous administration of gadolinium contrast $(0.4 \mathrm{ml} / \mathrm{kg})$. The following parameters were used: TR 8.9 msec; TE $1.8 \mathrm{msec}$; flip angle $20^{\circ}$; number of axial slices 232; slice thickness $1.4 \mathrm{~mm}$; number of averages 2; field of view $260.00 \mathrm{~mm}$; matrix size $256 \times 256$. The MR imaging data sets, which were acquired using the digital imaging and communication format, were transferred to a laptop personal computer. Using MRICro, a free software program available for use in MR image rendering, ${ }^{12}$ the 


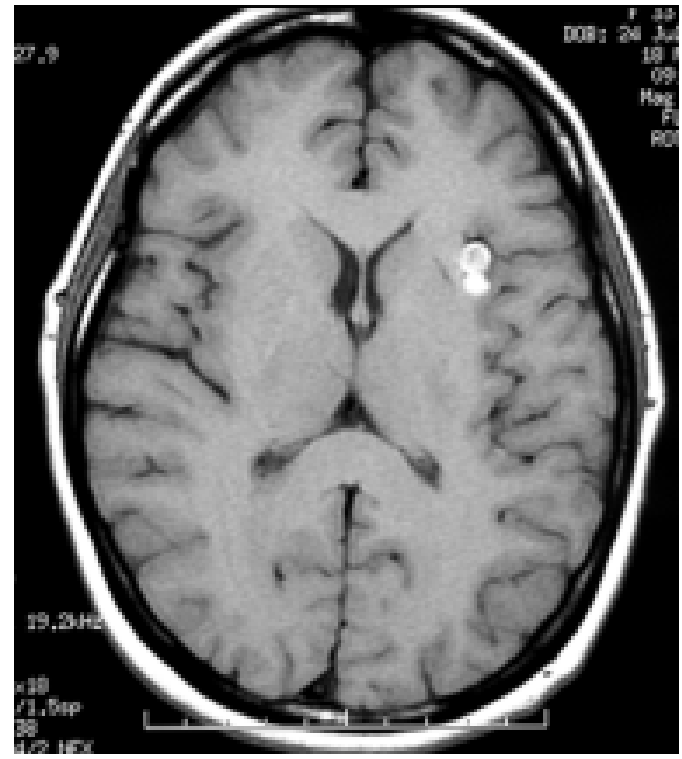

FIG. 1. Axial $\mathrm{T}_{1}$-weighted $\mathrm{MR}$ image demonstrating a cavernous hemangioma (according to postoperative diagnosis) of the left insular cortex.

MR images were reformatted into a 3D model. The same software allows one to isolate the brain from the overlying structures. The 3D reconstruction of the patient's brain can be grasped and rotated in accordance with the surgeon's needs. The lesion itself can be reconstructed and projected as a region of interest over the cortical surface or over the scalp.

In this case, the CM projected on a sulcus between the triangular and opercular parts of the inferior frontal gyrus (Broca area; Fig. 2). The projection of the region of interest was superimposed over the patient's skull and was used to design the craniotomy. After dural opening, a member of the surgical team obtained a digital photograph of the exposed cortex and-in the same surgical suitetransferred it to the laptop computer. Using common photoediting software, the intraoperative picture was cropped, rotated, and visually compared side by side with the 3D

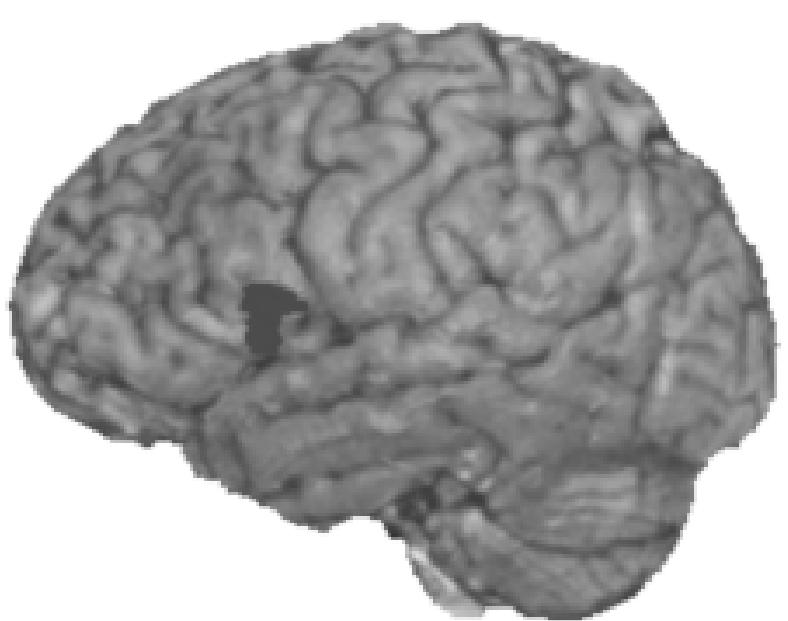

FIG. 2. A 3D rendering of the patient's MR image, including a surface projection of the lesion.

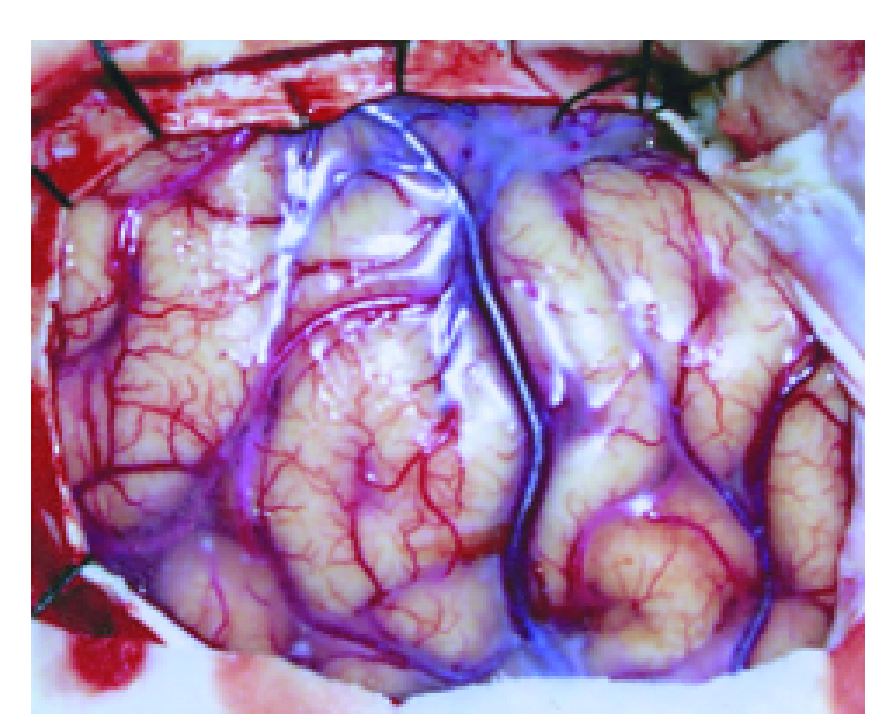

FIG. 3. Intraoperative surface matching. A digital photograph of the frontal cortex overlying the lesion (upper) is compared with a cropped and properly oriented reconstruction of the same area (lower). The sulcus between the triangular and opercular parts of the inferior frontal gyrus is located exactly over the lesion.

reconstruction of the same cortex (Fig. 3). The cortex overlying the lesion was identified by recognizing its morphological pattern. The angioma was quickly reached by splitting the sulcus between the triangular and opercular parts of the inferior frontal gyrus (Fig. 4). It was not necessary to dissect the sylvian fissure or to violate eloquent brain cortex.

Postoperative Course. A postoperative MR imaging study confirmed complete removal of the lesion (Fig. 5). Pathological examination confirmed the diagnosis of cavernous hemangioma. No neurological deficits were observed after surgery.

\section{Discussion}

Subcortical cavernomas involving critical brain areas are typical candidates for image-guided surgery. ${ }^{8,17}$ In the 


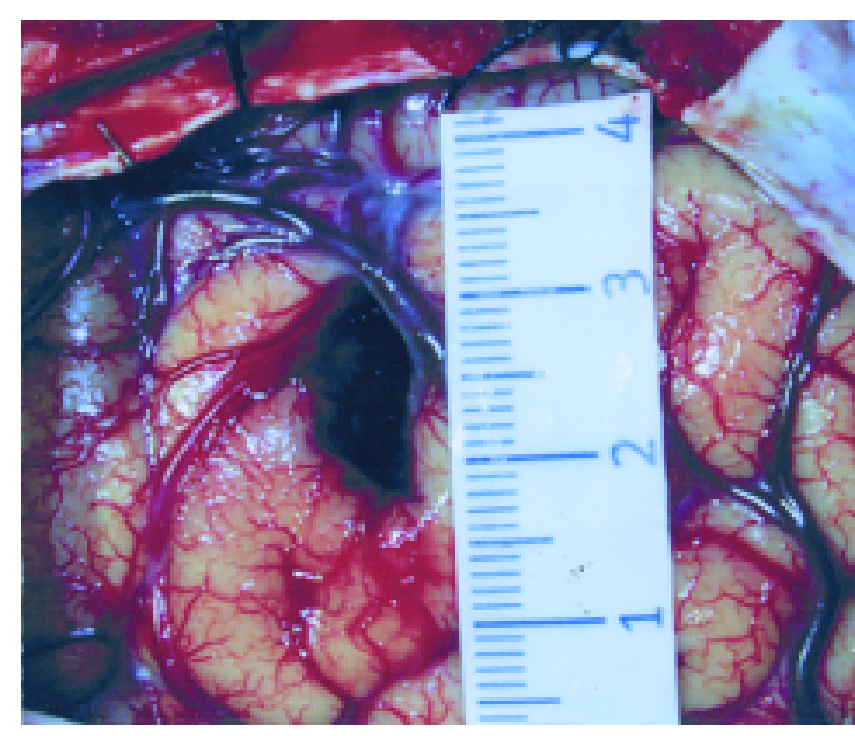

FIG. 4. Intraoperative photograph of the brain taken after a limited transsulcal approach was used to resect the $\mathrm{CM}$.

case reported here, MR imaging-based corticotopography proved to be a useful and accurate navigation method despite its simple technical requirements (a personal computer and an easily available software program). In addition, the technique has the advantage of being unaffected by brain shift, a phenomenon that must be taken into account when approaching such lesions with traditional navigation equipment. 5,10

In their surgical series of eight insular cavernous angiomas, Tirakotai and colleagues ${ }^{13}$ suggested that the impact of brain shift can be minimized if the patient is placed in the lateral position with the head kept horizontal. In this way, the loss of cerebrospinal fluid would displace the brain only in one direction, making the stereotactic guidance still reliable. Duffau and Fontaine ${ }^{7}$ used the same expedient to remove a left insular cavernoma with the aid of frameless neuronavigation.

Alternative techniques such as ultrasonography for localization of brain lesions have been advocated as reliable and relatively cost-effective methods of real-time navigation, to overcome the problem of brain shift. ${ }^{15,16}$ In fact, intraoperative ultrasonography requires dedicated equipment as well, does not provide the spatial resolution of MR imaging, and cannot be used to tailor the craniotomy.

The procedure we used is unaffected by the brain shift because the lesion is identified using anatomical markers, that is, sulci, gyri, and cortical vessels lying on the brain surface itself. These structures are strictly connected to the surgical target and move along with it, regardless of the brain displacement. In addition, the unequivocal, precise correlation between the sulcal anatomy and the surgical target as defined by MR imaging-based corticotopography allowed us to choose the most direct approach to the involved portion of the insula, that is, a frontal transsulcal route, thus avoiding the need for a classic transsylvian approach. Therefore, the lenticulostriate arteries were not manipulated. Also, by using a transsulcal approach, we can reach lesions arising in eloquent cortical areas through

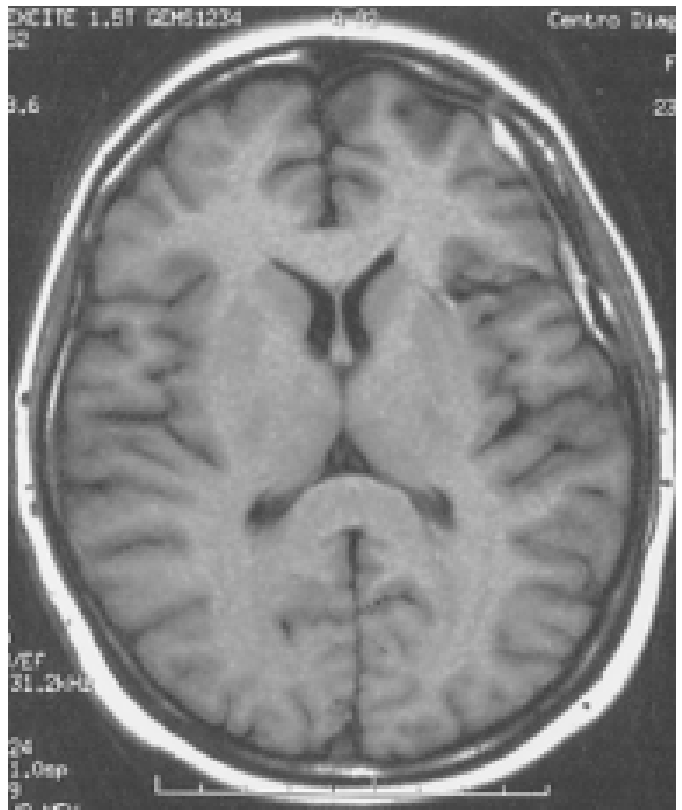

FIG. 5. Postoperative axial MR image confirming removal of the lesion. The narrow surgical pathway centered on the lesion site is still visible.

the depth of the sulcus, with limited brain retraction and keeping to a minimum the degree of tissue loss.

\section{Conclusions}

This case illustrates how, for selected brain lesions, MR imaging-based corticotopography can provide a viable, inexpensive alternative to traditional neuronavigation. These features might encourage its use in subcortical lesions requiring intraoperative image guidance. The main limitation of the technique is the loss of accuracy for lesions deeper than 2.5 to $3 \mathrm{~cm}$. In these cases, the surgical trajectory chosen between the surface marker and the targeted lesion would be subject to an inherent risk of error.

\section{References}

1. Aiba T, Tanaka R, Koike T, Kameyama S, Takeda N, Komata T: Natural history of intracranial cavernous malformations. J Neurosurg 83:56-59, 1995

2. Amin-Hanjani S, Ogilvy CS, Ojemann RG, Crowell RM: Risks of surgical management for cavernous malformations of the nervous system. Neurosurgery 42:1220-1228, 1998

3. Bertalanffy H, Benes L, Miyazawa T, Alberti O, Siegel AM, Sure U: Cerebral cavernomas in the adult. Review of the literature and analysis of 72 surgically treated patients. Neurosurg Rev 25:1-55, 2002

4. Bertalanffy H, Gilsbach JM, Eggert HR, Seeger W: Microsurgery of deep-seated cavernous angiomas: report of 26 cases. Acta Neurochir 108:91-99, 1991

5. Dorward NL, Alberti O, Palmer JD, Kitchen ND, Thomas DG: Accuracy of true frameless stereotaxy: in vivo measurement and laboratory phantom studies. Technical note. J Neurosurg 90:160-168, 1999

6. Duffau H, Capelle L, Lopes M, Faillot T, Sichez JP, Fohanno D: The insular lobe: physiopathological and surgical considerations. Neurosurgery 47:801-811, 2000 
7. Duffau H, Fontaine D: Successful resection of a left insular cavernous angioma using neuronavigation and intraoperative language mapping. Acta Neurochir (Wien ) 147:205-208, 2005

8. Gralla J, Ganslandt O, Kober H, Buchfelder M, Fahlbusch R, Nimsky C: Image-guided removal of supratentorial cavernomas in critical brain areas: application of neuronavigation and intraoperative magnetic resonance imaging. Minim Invasive Neurosurg 46:72-77, 2003

9. Heffez DS: Stereotactic transsylvian, transinsular approach for deep-seated lesions. Surg Neurol 48:113-124, 1997

10. Roberts DW, Hartov A, Kennedy FE, Miga MI, Paulsen KD: Intraoperative brain shift and deformation: a quantitative analysis of cortical displacement in 28 cases. Neurosurgery 43: 749-760, 1998

11. Roper SN, Levesque MF, Sutherling WW, Engel J Jr: Surgical treatment of partial epilepsy arising from the insular cortex. Report of two cases. J Neurosurg 79:266-269, 1993

12. Rorden C, Brett M: Stereotaxic display of brain lesions. Behav Neurol 12:191-200, 2000

13. Tirakotai W, Sure U, Benes L, Krischek B, Bien S, Bertalanffy $\mathrm{H}$ : Image-guided transsylvian, transinsular approach for insular cavernous angiomas. Neurosurgery 53:1299-1305, 2003
14. Ture U, Yaşargil DC, Al-Mefty O, Yaşargil MG: Topographic anatomy of the insular region. J Neurosurg 90:720-733, 1999

15. Unsgaard G, Ommedal S, Muller T, Gronningsaeter A, Nagelhus Hernes TA: Neuronavigation by intraoperative threedimensional ultrasound: initial experience during brain tumor resection. Neurosurgery 50:804-812, 2002

16. Woydt M, Horowski A, Krone A, Soerensen N, Roosen K: Localization and characterization of intracerebral cavernous angiomas by intra-operative high-resolution color-duplexsonography. Acta Neurochir 141:143-152, 1999

17. Zamorano L, Matter A, Saenz A, Buciuc R, Diaz F: Interactive image-guided resection of cerebral cavernous malformations. Comput Aided Surg 2:327-332, 1997

Manuscript received May 10, 2006.

Accepted in final form May 30, 2006.

Address reprint requests to: Sergio Paolini, M.D., Via G.A. Sartorio 44, 00146 Roma, Italia. email: spao2@yahoo.com. 